\title{
Peripheral Nerve Stimulation of the Saphenous and Superior Lateral Genicular Nerves for Chronic Pain After Knee Surgery
}

\author{
Ahish Chitneni ${ }^{1}$, Amnon A. Berger ${ }^{2}$, Vwaire Orhurhu ${ }^{3}$, Alan D. Kaye ${ }^{4}$, Jamal Hasoon ${ }^{5}$ \\ 1 School of Osteopathic Medicine in Arizona, Mesa, AZ, A.T. Still University, ${ }^{2}$ Beth Israel Deaconess Medical Center, Anesthesiology, Critical Care, and \\ Pain Medicine, Harvard Medical School, Boston, MA, ${ }^{3}$ Department of Anesthesia, Critical Care and Pain Medicine, Massachusetts General Hospital \\ and Harvard Medical School, Boston, MA, ${ }^{4}$ Department of Anesthesiology, Louisiana State University Health Shreveport, Shreveport, LA, 5 Beth Israel \\ Deaconess Medical Center, Anesthesiology, Critical Care, and Pain Medicine, Harvard Medical School, Boston, MA; Pain Specialists of America, Austin, \\ TX
}

Keywords: peripheral nerve stimulation, chronic pain, knee pain, tka

https://doi.org/10.52965/001c.24435

Orthopedic Reviews

Vol. 13, Issue 1, 2021

\begin{abstract}
Total knee arthroplasty (TKA) is one of the most commonly conducted surgeries in the United States. Typically, TKA is conducted to relieve pain from patients with long-standing osteoarthritis. Postoperative knee pain is a common issue after TKA. For some patients, postoperative knee pain exceeds the normal 3-6-month phase and becomes chronic. Pain is typically managed with the use of medications and physical therapy. In this case, we describe the use of peripheral nerve stimulation (PNS) of the saphenous and superior lateral genicular nerves for a patient experiencing chronic postoperative knee pain utilizing SPRINT PNS technology.
\end{abstract}

\section{INTRODUCTION}

Total knee arthroplasty (TKA) is one of the most commonly conducted surgeries in the United States. The total prevalence of TKA by age 80 in the population was $10 \%$ with prevalence higher among women than men. ${ }^{1}$ Over 5 million individuals receive a TKA in the United States each year. ${ }^{1}$ In general, the procedure is conducted on patients with severe osteoarthritis $(\mathrm{OA})$ that is refractory to conservative treatment. Conservative management of OA typically consists of pain control with the use of NSAIDs or acetaminophen and physical therapy which can control progression of the condition and prevent the need for surgical intervention. ${ }^{2}$ In many cases, conservative therapy and pharmacological options are unable to resolve knee pain, and further intervention may become necessary. Some common interventions include administration of intra-articular corticosteroid injections. Past research has concluded that corticosteroid injections for the management of knee pain in osteoarthritis is variable and typically only provides pain relief for a few weeks. ${ }^{3}$ In addition, the use of corticosteroid injections should be limited to once every three months. Therefore, when patients become refractory to conservative treatment, a TKA is typically conducted to relieve pain and progression of the condition.

TKA is typically conducted in patients with symptomatic osteoarthritis in a minimum of two of the three components of the knee. ${ }^{4}$ In patients with symptomatic osteoarthritis in only 1 component of the knee, a partial knee arthroplasty (PKA) can be considered. The goal of the procedure is to provide pain relief for patients experiencing symptoms from osteoarthritis.

Despite the goal of the procedure to provide pain relief, in many cases patients experience postoperative chronic knee pain after surgery. Per the International Association for the Study of Pain (IASP), chronic pain is defined as pain that lasts more than 3 months. ${ }^{5}$ In general, post-operative pain after a TKA subsides after 3-6 months. ${ }^{6}$ Therefore, patients experiencing pain for greater than 6 months postoperatively, can be defined as experiencing chronic pain. Though medication management is a typical treatment for postoperative pain, various other treatments such a genicular nerve block and peripheral nerve stimulation for chronic knee pain have shown promising results. ${ }^{7,8}$

Peripheral nerve stimulation (PNS) is a pain management procedure that uses electrical currents to target specific nerves. Research has shown that PNS is effective in the treatment of a variety of chronic pain conditions. Typically, PNS consists of an electrode that is implanted next to the nerve of choice, which can then be stimulated with the goal of providing pain relief.

In this case, we present the use of PNS targeting the superior lateral genicular nerve and the saphenous nerve for a patient with severe postoperative knee pain after TKA. We chose to utilize a temporary 60-day PNS system. (SPRINT PNS System [SPR Therapeutics, Inc., Cleveland, Ohio]). This device is implanted for 60 days and then removed at the end of therapy with the goal of achieving longstanding pain relief.

\section{CASE PRESENTATION}

The patient was a 73-year-old female with a history of chronic knee pain after TKR on her left knee 10 months prior. The patient noted that her pain was burning and stabbing in nature. She reported a pain score of 10/10 intensity on numerical rating scale. She tried conservative management with physical therapy, acetaminophen, nonsteroidal 
anti-inflammatories, membrane stabilizers, and opioids. Even with opioid therapy the patient averaged a pain score of $8 / 10$ intensity and reported significant pain and impaired functionality. The patient was offered PNS utilizing the SPRINT PNS system and agreed to pursue this therapy.

The patient was positioned supine, and the left leg was prepped and draped in a sterile fashion. Utilizing a $12 \mathrm{MHz}$ linear ultrasound transducer, the saphenous nerve was identified and marked. The saphenous nerve is a sensory branch of the femoral nerve that supplies sensory innervation over the medial knee as well as infrapatellar branches to the knee joint. The PNS lead was placed near the saphenous nerve and secured after satisfactory paresthesia testing. The superior lateral genicular nerve was then targeted utilizing fluoroscopy. A lateral fluoroscopic view was obtained, and the introducer needle was advanced into the region of the superior lateral genicular nerve. The lead was repositioned until a satisfactory paresthesia was reported over the lateral aspect of the knee. The leads were then secured without complications.

The patient reported significant pain relief 3 days into her trial. She noted improvement in activity and functionality as she was now able to do outdoor activities and do her normal activities of daily living without having to take medications. She stated her pain was improved $80 \%$ after 1 month of therapy. After 2 months of therapy the patient presented for her follow up and reported 90\% improvement in her pain scores. Additionally, she had discontinued all pain medications. Her leads were subsequently removed without complications. The patient has continued to report significant improvement in pain and functionality 2 months after lead removal and is still off all pain medications.

\section{DISCUSSION}

Given the traditional use of opioids and medication management for treatment of chronic post-operative knee pain, there exists a need for research on the use of alternative methods for pain relief in patients who undergo TKA. Our literature search led to several research studies that used PNS for pain relief postoperatively after a TKA. One study conducted by Ilfeld et. al observed the use of percutaneous peripheral nerve stimulation for the treatment of postoper- ative pain following total knee arthroplasty. ${ }^{9}$ In the study, seven subjects underwent treatment with ultrasound guided PNS postoperatively with stimulation delivered to the femoral and sciatic nerves. Results from the study showed that in six of the seven subjects, the average daily pain scores the first two weeks was rated as $<4$ and a large majority of the subjects discontinued opioid use within the first week postoperatively. Given the positive results from the study in reducing pain and opioid usage, the use of PNS should be strongly considered early in the postoperative course.

Although not conducted after a TKA, another study by Ilfred et. al observed the use of PNS of the femoral nerve for postoperative analgesia following anterior cruciate ligament (ACL) reconstruction. In this study, patients were randomized to receive PNS or sham for 5 minutes. ${ }^{10}$ Overall, results showed that the use of PNS was able to provide adequate pain relief and reduced opioid usage in patients undergoing ACL reconstruction. The importance of this study is to demonstrate the utility of PNS for a variety of knee surgeries and should not be limited to patients undergoing TKA.

\section{CONCLUSION}

Although postoperative knee pain after TKA can be typical in the short term, many patients experience chronic knee pain after surgical intervention. Chronic knee pain postoperatively has been traditionally managed medically with opioids or other pain medications. Given the prevalence of postoperative chronic knee pain, alternative non-opioid interventions should be utilized earlier in the treatment algorithms to provide patients with an alternative form of pain management. Our case demonstrates the utility of peripheral nerve stimulation targeting the superior lateral genicular nerve and the saphenous nerve for a patient with chronic postoperative knee pain after TKA. We believe that PNS is a viable option for postoperative chronic pain complaints and should be considered before resorting to opioid therapy for chronic knee pain after surgery.

Submitted: May 01, 2021 EST, Accepted: May 21, 2021 EST 


\section{REFERENCES}

1. Kremers HM, Larson DR, Crowson CS, et al. Prevalence of total hip and knee replacement in the United States. J Bone Jt Surg Am. 2015;97:1386-1397. doi:10.2106/ibjs.n.01141

2. Sarzi-Puttini P, Cimmino MA, Scarpa R, et al. Osteoarthritis: an overview of the disease and its treatment strategies. Semin Arthritis Rheum. 2005;35(1 Suppl 1):1-10. doi:10.1016/j.semarthrit.200 $\underline{5.01 .013}$

3. Bellamy N, Campbell J, Robinson V, Gee T, Bourne $\mathrm{R}$, Wells $\mathrm{G}$. Intraarticular corticosteroid for treatment of osteoarthritis of the knee. Cochrane Database Syst Rev. Published online April 19, 2006:CD005328. doi:1 0.1002/14651858.cd005328.pub2

4. Hsu H, Siwiec RM. Knee Arthoplasty. In: StatPearls. StatPearls Publishing; 2020. Accessed January 2021. $\underline{\mathrm{h}}$ ttps://www.ncbi.nlm.nih.gov/books/NBK507914/

5. Wylde V, Beswick A, Bruce J, Blom A, Howells N, Gooberman-Hill R. Chronic pain after total knee arthroplasty. EFORT Open Rev. 2018;3(8):461-470. do i:10.1302/2058-5241.3.180004
6. Lenguerrand E, Wylde V, Gooberman-Hill R, et al. Trajectories of Pain and Function after Primary Hip and Knee Arthroplasty: The ADAPT Cohort Study. PLoS ONE. 2016;11(2):e0149306. doi:10.1371/journa l.pone.0149306

7. Sahoo RK, Krishna C, Kumar M, Nair AS. Genicular nerve block for postoperative pain relief after total knee replacement. Saudi J Anaesth. 2020;14:235-237. doi:10.4103/sja.sja_611_19

8. Hasoon J, Chitneni A, Urits I, Viswanath O, Kaye AD. Peripheral Stimulation of the Saphenous and Superior Lateral Genicular Nerves for Chronic Knee Pain. Cureus. 2021;13(4):e14753. doi:10.7759/cureu $\underline{\text { s.14753 }}$

9. Ilfeld BM, Ball ST, Gabriel RA, et al. A feasibility study of percutaneous peripheral nerve stimulation for the treatment of postoperative pain following total knee arthroplasty. Neuromodulation. 2019;22:653-660. doi:10.1111/ner.12790

10. Ilfeld BM, Said ET, Finneran JJ 4th, et al. Ultrasound - Guided Percutaneous Peripheral Nerve Stimulation: Neuromodulation of the Femoral Nerve for Postoperative Analgesia Following Ambulatory Anterior Cruciate Ligament Reconstruction: A Proof of Concept Study. Neuromodulation. 2019;22(5):621-629. doi:10.1111/ner.12851 